\title{
Synergistic effect of celecoxib in tumor necrosis factor-related apoptosis-inducing ligand treatment in osteosarcoma cells
}

\author{
GONG LIU, MING-YANG YU, XU HUANG, DONG ZHU, SHIHUAN CHENG, RENSHI MA and GUISHAN GU \\ Department of Orthopaedics, The First Hospital of Jilin University, Changchun, Jilin 130021, P.R. China
}

Received November 11, 2013; Accepted June 6, 2014

DOI: $10.3892 / \mathrm{mmr} .2014 .2409$

\begin{abstract}
Tumor necrosis factor-related apoptosis-inducing ligand (TRAIL) is under clinical development as a cancer therapeutic as it has been shown to induce apoptosis in numerous types of cancer cells without significant toxicity towards normal cells. However, the majority of osteosarcoma (OS) tumors are resistant to TRAIL. Thus, the development of cancer therapeutics that overcome TRAIL resistance is required.In the present study, celecoxib (CXB), a non-steroidal anti-inflammatory drug, was administered in combination with TRAIL to induce cell apoptosis and the doses of the two drugs were simultaneously reduced. The effects of this combination treatment were examined in MG-63 human OS cancer cell lines in culture. Assays of proliferation, apoptosis and tumor growth were performed, along with analysis of the proteins involved. The results revealed that CXB sensitized TRAIL-resistant MG-63 OS cells to TRAIL-induced apoptosis through downregulation of cellular B-cell lymphoma 2 (Bcl-2), Bcl-2-associated X protein, caspase-8 and caspase-3. Furthermore, combination treatment reduced tumor growth in a nude rat model. In conclusion, the experimental results provided evidence that the combined administration of $\mathrm{CXB}$ and TRAIL is potentially a novel treatment method of OS tumors.
\end{abstract}

\section{Introduction}

The most frequent malignant bone tumor in children and adolescents, osteosarcoma (OS) (1), is a devastating disease. OS typically exhibits marked local aggressiveness and shows a tendency to metastasize to distant bones and the lungs. The administration of neoadjuvant chemotherapy and improvements in surgical technology have increased the OS survival rate to $65-75 \%$; however, pulmonary metastases occur in

Correspondence to: Professor Guishan Gu, Department of Orthopaedics, The First Hospital of Jilin University, 71 Xinmin Street, Chaoyang, Changchun, Jilin 130021, P.R. China E-mail: guguisan1110@126.com

Key words: osteosarcoma, celecoxib, tumor necrosis factor-related apoptosis-inducing ligand, apoptosis
$40-50 \%$ of patients with OS and remain a predominant cause of fatalities (2-4). Thus, novel drugs and treatment methods for OS are urgently required.

Tumor necrosis factor-related apoptosis-inducing ligand (TRAIL) is a promising antitumor agent since it is capable of killing tumor cells via receptor-mediated apoptosis $(5,6)$. Preclinical studies using recombinant TRAIL (rhTRAIL) have provided evidence for exogenous TRAIL efficacy in suppressing tumor growth in vitro and in vivo (7-9). However, resistance towards TRAIL may result in failure at any step in the death signaling cascade. For example, TRAIL resistance may occur at the receptor level, due to deregulated expression, or at the death-induced signaling complex (DISC) level, mediated by proteins counteracting DISC formation (10-12). In addition, an inability to activate mitochondria during apoptosis, due to high expression levels of antiapoptotic proteins, may result in TRAIL resistance $(13,14)$. Furthermore, antiapoptotic signaling pathways, including the phosphoinositol-3-kinase/Akt signaling pathway, are aberrantly activated in numerous types of tumor cell, therefore contributing to TRAIL resistance $(15,16)$. Thus, identifying cancer therapeutic agents that overcome TRAIL resistance is required.

Celecoxib (CXB) is a cyclooxygenase 2-selective nonsteroidal anti-inflammatory drug, which has been approved for the treatment of adult arthritis. CXB has also been shown to exhibit therapeutic benefits in various types of cancer (17). Currently, CXB is being widely investigated in clinical trials for therapeutic activity against various cancer types as a single agent and in combination with other agents $(18,19)$.

The aim of the present study was to evaluate the potency of CXB in combination with TRAIL in inhibiting OS cancer cell growth and proliferation, and to reveal the underlying molecular mechanisms involved in TRAIL-induced apoptosis. Whether CXB, as an adjuvant agent, allows the effective dosage of TRAIL required to treat OS cancer cells to be reduced was also determined.

\section{Materials and methods}

Cell lines. The MG-63 human OS cell line was obtained from the American Type Culture Collection (Manassas, VA, USA). The cells were cultured immediately following unfreezing at a concentration of $5 \times 10^{6}$ cells $/ \mathrm{ml}$ in Dulbecco's modified Eagle's medium (DMEM; Hyclone, Logan, UT, 
USA) supplemented with $10 \%$ fetal bovine serum (FBS; Hyclone), $100 \mathrm{U} / \mathrm{ml}$ penicillin and $100 \mu / \mathrm{ml}$ streptomycin (Gibco Life Technologies, Carlsbad, CA, USA). The cells were incubated in a humidified atmosphere containing 5\% carbon dioxide at $37^{\circ} \mathrm{C}$.

Reagents. CXB (Sigma-Aldrich, St. Louis, MO, USA) was dissolved in dimethyl sulfoxide (DMSO; Sigma-Aldrich) to create a $1 \mathrm{mM}$ stock solution, stored at $-20^{\circ} \mathrm{C}$ and diluted in fresh medium immediately prior to use. TRAIL (with Enhancer applied at a concentration of $1 \mu \mathrm{g} / \mathrm{ml}$ ) was purchased from Alexis Biochemicals (San Diego, CA, USA). For western blot analysis, the following antibodies were used: Mouse monoclonal anti- $\beta$-actin (Sigma-Aldrich); mouse monoclonal anti-B-cell lymphoma 2 (Bcl-2) and anti-survivin, and horseradish peroxidase-conjugated anti-mouse $\operatorname{IgG}$ (Santa Cruz Biotechnology, Inc., Santa Cruz, CA, USA). Nonidet P-40 lysis buffer and MTT were obtained from Sigma-Aldrich. MTT solution was prepared by dissolving $1 \mathrm{mg}$ of compound in $1 \mathrm{ml}$ phosphate-buffered saline (PBS). The solution was protected from light, stored at $4^{\circ} \mathrm{C}$ and used within one month.

Cell viability assay. MG-63 cells grown in monolayers were harvested and dispensed in 96-well culture plates in $100 \mu \mathrm{l}$ DMEM at a concentration of $5 \times 10^{3}$ cells per well. After $24 \mathrm{~h}$, differential drug concentrations of TRAIL $(0-200 \mathrm{ng} / \mathrm{ml})$, CXB $(0-250 \mu \mathrm{M})$ or a combination $(0-100 \mathrm{ng} / \mathrm{ml}$ TRAIL plus $30 \mu \mathrm{M}$ CXB) were added to the cells. Cell viability after 24 , 48 and 72-h incubation was measured using the MTT colorimetric assay with an ELISA microplate reader (Thermo Labsystems, Helsinki, Finland) at $490 \mathrm{~nm}$ with minor modifications according to methods described in a previous study (20). The inhibition rate was calculated according to the following formula: Inhibition rate $(\%)=[1-($ average absorbance of experimental group/average absorbance of blank control group)] x 100.

Detection of apoptosis. MG-63 cells were cultured in six-well plates in DMEM with 10\% FBS medium and were treated with the respective half maximal inhibitory concentrations $\left(\mathrm{IC}_{50}\right)$ of TRAIL, CXB or their combination for $48 \mathrm{~h}$. The cover slips were washed three times with PBS and single cell suspensions were fixed in $1 \%$ PBS. The cells were stained with $100 \mu \mathrm{g} / \mathrm{ml}$ acridine orange (Sigma-Aldrich) and $100 \mu \mathrm{g} / \mathrm{ml}$ ethidium bromide for $1 \mathrm{~min}$. Subsequently, the cells were observed under a fluorescent microscope (IX73-U; Olympus, Tokyo, Japan). At least 200 cells were counted and the percentage of apoptotic cells was determined. All experiments were performed in triplicates conducted on five occasions. Caspase activation, Bax-2 and Bcl-2 expression levels were also detected by western blotting as an additional indicator of apoptosis.

Western blot analysis. MG-63 cells $\left(1 \times 10^{6}\right.$ cells per $100 \mathrm{~mm}$ plate) were treated with TRAIL, CXB or their combination at the respective $\mathrm{IC}_{50}$ doses for $24 \mathrm{~h}$. The cells in control wells were treated with $0.1 \%$ DMSO for $1 \mathrm{~h}$. All cells were activated with recombinant human epidermal growth factor $(25 \mathrm{ng} / \mathrm{ml}$; Sigma-Aldrich) for $30 \mathrm{~min}$. The cells were then scraped and lysed in Nonidet P-40 lysis buffer. Cell extracts (50 $\mu \mathrm{g}$ protein) were separated by SDS-PAGE and transferred to nitrocellulose membranes, which were blocked in 3\% bovine serum albumin (BSA) for $2 \mathrm{~h}$. Subsequent to blocking, the membranes were incubated with primary antibodies $\beta$-actin $(1: 5,000)$, Bcl-2 $(1: 1,000)$ and survivin $(1: 3,000)$ overnight at $4^{\circ} \mathrm{C}$ and then with horseradish peroxidase-conjugated anti-mouse $\operatorname{IgG}(1: 10,000)$ for $2 \mathrm{~h}$ at room temperature. The proteins were visualized by exposing the chemiluminescence substrate (Sigma-Aldrich) to X-OMAT AR autoradiography film (Eastman Kodak, Rochester, NY, USA).

Animal experiments. The tumor response to TRAIL and CXB treatment was investigated using the MG-63 tumor-bearing female BALB mouse model (obtained from the Experimental AnimalCenter of Jilin University, Changchun, China). The mice were maintained in accordance with the Institute of Animal Ethics Committee guidelines approved by the University of Jilin Animal Care and Use Committee, Changchun, China. The present study was approved by the ethics committee of the First Hospital of Jilin University (Changchun, China) The mice were housed and acclimated in a pathogen-free environment at the animal facility of the First Hospital of Jilin University for one week prior to injection with tumor cells.

Exponentially growing MG-63 cells were harvested and a tumorigenic dose of $2.5 \times 10^{6}$ cells was injected intraperitoneally into the 6 to 7 week-old female BALB mice. Tumors were allowed to grow in the mice for seven days, then the animals were randomly assigned to one of four treatment groups (10 mice per group). The control group received $1 \%$ polysorbate resuspended in deionized water. The other three groups were treated with CXB $(3.7 \mathrm{mg} / \mathrm{kg}$ body weight $)$, TRAIL ( $90 \mathrm{mg} / \mathrm{kg}$ body weight) or CXB plus TRAIL (2 and $50 \mathrm{mg} / \mathrm{kg}$ body weight, respectively) intraperitoneally on alternative days for two weeks. The doses were selected as determined by previous experiments $(21,22)$. Tumor weight and tumor volume were measured prior to administration of the treatment injections and on days 7 and 14 of treatment. On day 15, the animals were sacrificed using chloroform and spleen tissues were collected and cultured for a splenocyte surveillance study.

Assay of splenocyte proliferation. Single-cell spleen suspensions were generated and pooled in serum-free DMEM by filtering the suspension through a sieve mesh with the aid of a glass homogenizer, which exerted gentle pressure on the spleen fragments. The samples were washed twice in PBS with $0.1 \%(\mathrm{w} / \mathrm{v})$ BSA Following centrifugation at $200 \mathrm{xg}$ for $10 \mathrm{~min}$, the cells were seeded at $3 \times 10^{3}$ cells per well in 96-well flat-bottomed microplates in triplicate in DMEM supplemented with $10 \%$ FBS. The cells were incubated in a total volume of $100 \mu \mathrm{l}$ per well. Serum-free DMEM served as a control. After $24 \mathrm{~h}$, cell proliferation was measured using an MTT assay.

Statistical analysis. All statistical analyses were performed using GraphPad Prism 5 software (La Jolla, CA, USA). Values are presented as mean \pm standard deviation. Statistical significance was determined using one-way analysis of variance. $\mathrm{P}<0.001$ and $\mathrm{P}<0.05$ were considered to indicate statistically significant differences. 


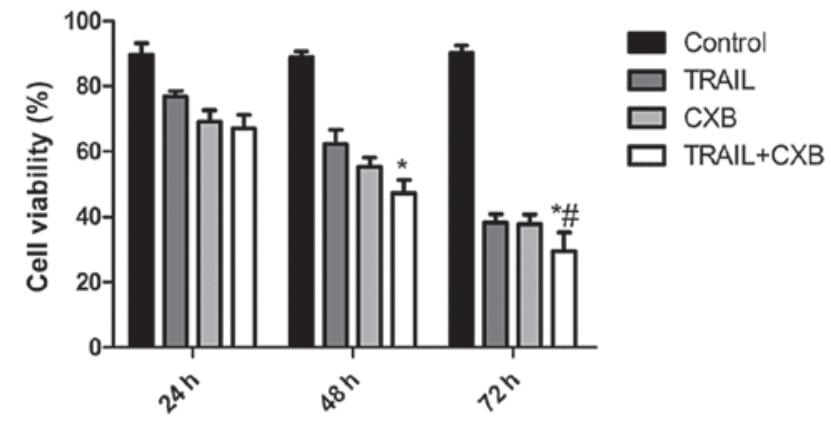

Figure 1. Effects of CXB, TRAIL and TRAIL+CXB combination treatment on MG-63 osteosarcoma cell growth inhibition. Cell viability was determined by the MTT method. The assay was performed in triplicate. Inhibition of cell growth was observed after 24,48 and $72 \mathrm{~h}$. ${ }^{*} \mathrm{P}<0.05$ vs. $24 \mathrm{~h} ;{ }^{\#} \mathrm{P}<0.05$ vs. $\mathrm{CXB}$, one-way analysis of variance. CXB, celecoxib; TRAIL, tumor necrosis factor-related apoptosis-inducing ligand.

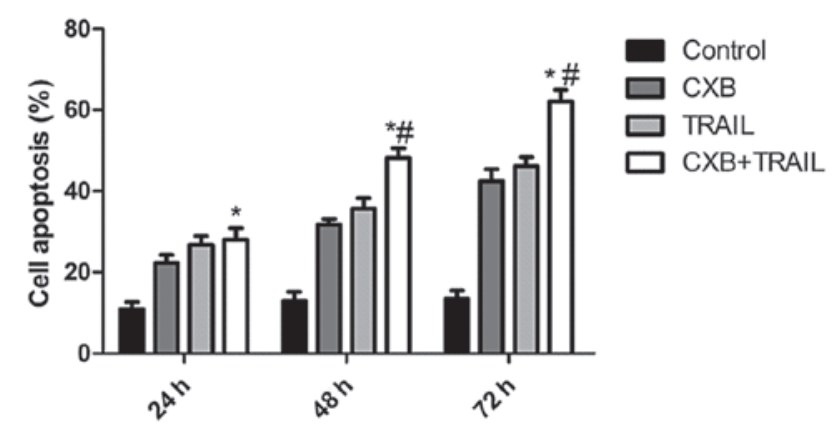

Figure 2. Effect of CXB, TRAIL and TRAIL+CXB combination treatment on MG-63 osteosarcoma cell apoptosis in vitro. This assay was performed in triplicate. Increased cell apoptosis was observed after 24, 48 and $72 \mathrm{~h}$ drug treatment. $\mathrm{P}<0.05$ vs. $24 \mathrm{~h} ;{ }^{\#} \mathrm{P}<0.05$ vs. CXB. CXB, celecoxib; TRAIL, tumor necrosis factor-related apoptosis-inducing ligand.

\section{Results}

CXB enhances TRAIL-induced OS cancer cell viability. To determine the effect of TRAIL, CXB and the combination treatment on the viability of OS cancer cells in vitro, MG-63 cells were treated with increasing concentrations of $\mathrm{CXB}$ (0-200 $\mu \mathrm{M})$ or TRAIL $(0-200 \mathrm{ng} / \mathrm{ml})$. The $\mathrm{IC}_{50}$ value of TRAIL alone was determined to be $100.18 \pm 5.28 \mathrm{ng} / \mathrm{ml}$, and the $\mathrm{IC}_{50}$ value of $\mathrm{CXB}$ alone was $100.5 \pm 0.780 \mu \mathrm{M}$. Combination treatment $(0-100 \mathrm{ng} / \mathrm{ml}$ TRAIL in the presence of $30 \mu \mathrm{M}$ $\mathrm{CXB})$ resulted in a shift of the cell viability curve towards lower drug concentrations such that the $\mathrm{IC}_{50}$ value of TRAIL was reduced to $50.76 \pm 5.14 \mathrm{ng} / \mathrm{ml}$. These results indicated that treatment with the two agents was more cytotoxic than either alone. According to the results, respective drug $\mathrm{IC}_{50}$ values were selected for further treatments throughout the study. In addition, the inhibitory rates in the combination treatment group were found to be higher than those of the single drug groups. No significant difference was detected between the TRAIL and CXB single treatment groups (Fig. 1).

CXB enhances TRAIL-induced apoptosis. The effects of TRAIL and CXB on MG-63 cell apoptosis were then analyzed. MG-63 cells ( $\mathrm{IC}_{50}$ values: $100 \mu \mathrm{M}$ for $\mathrm{CXB}$ and $100 \mathrm{ng} / \mathrm{ml}$ for TRAIL) treated with either TRAIL or CXB exhibited an increased percentage of apoptotic cells compared with untreated cells (Fig. 2). However, the low-dose combination (30 $\mu \mathrm{M}$ CXB plus $50 \mathrm{ng} / \mathrm{ml}$ TRAIL) resulted in an even greater percentage of apoptotic cells than the higher doses of either drug alone. These data are consistent with the results from the MTT assay, which indicated an additive mechanism of TRAIL and CXB in inducing cell death through apoptosis.

TRAIL and CXB treatment results in significant inhibition of tumor growth. The in vivo therapeutic efficacy of TRAIL and CXB was assessed in female BALB mice bearing MG-63 cell tumors. TRAIL and CXB each induced tumor regression and inhibited tumor growth in the respective treatment groups. The mice were subsequently sacrificed on day 21 after treatment and tumor tissue was excised. The tumor weight of the animals was subsequently measured; the average tumor weight in the treatment groups was lower than that in the untreated group. Furthermore, the average tumor weight in the combination group was significantly lower than that in the single-treatment CXB or TRAIL groups $(\mathrm{P}<0.01$; Fig. $3 \mathrm{~A})$. In addition, the tumor volume was measured on days 7,14 and 21 of treatment. The tumor volume in the treatment groups increased at a slower rate than that in the untreated group. The tumor volume in the combination treatment group increased at a significantly slower rate than that in either CXB or TRAIL single treatment groups $(\mathrm{P}<0.01$; Fig. 3B). These result demonstrated that TRAIL and CXB, and particularly their combination, induced tumor regression and inhibited tumor growth.

CXB increases TRAIL-induced splenocyte proliferation inhibition. To assess the efficacy of TRAIL and CXB in modulating splenocyte proliferation, spleen cells from treated mice with MG-63 cell tumors were isolated and cultured in DMEM supplemented with $10 \% \mathrm{FBS}$ for $24 \mathrm{~h}$ and subjected to in vitro proliferation assays using the MTT method. As shown in Fig. 4, the inhibitory rate in the combination treatment group was higher than that in the single drug treatment groups, which demonstrated that combination treatment inhibited OS cell proliferation.

Preliminary clarification of the mechanism of $C X B$ - and TRAIL-induced induction of apoptosis. To clarify the molecular mechanisms of CXB-TRAIL combination treatment on the induction of OS cell apoptosis, the effects of CXB and TRAIL on the activation of proteins Bcl-2 and survivin, which are involved in cell apoptosis, were analyzed. As shown in Fig. 5, CXB administered in combination with TRAIL significantly inhibited Bcl-2 and survivin expression levels in OS tumor cells compared with the single treatment and untreated groups. These results indicated that $\mathrm{CXB}$ and TRAIL combination therapy inhibited apoptosis in OS tumor cells.

\section{Discussion}

In the present study, combining TRAIL with CXB significantly induced cell apoptosis and inhibited OS tumor growth, whereas single treatment did not exert a significant effect. Although the two compounds have each been extensively investigated, to the best of our knowledge, this study is the 
A

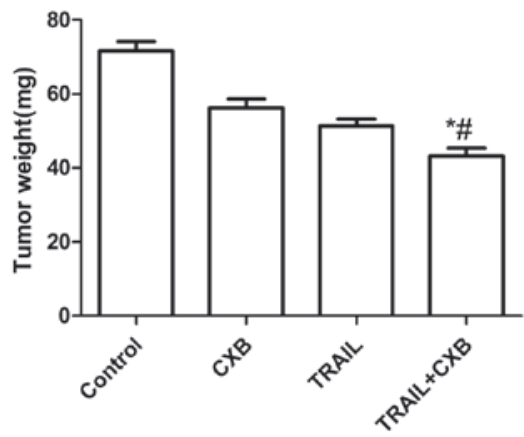

B

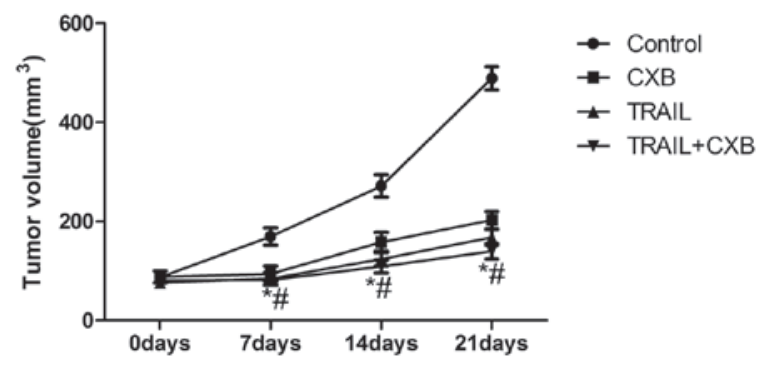

Figure 3. Antitumor activity of TRAIL and CXB in BALB/c mice bearing MG-63 osteosarcoma cell tumors. (A) Tumor weight in treated and untreated mice after 21 days. (B) Tumor volume in treated and untreated mice on days $0,7,14$ and 21 . "P<0.05 vs. untreated mice. ${ }^{~} \mathrm{P}<0.05$ vs. $\mathrm{CXB}$-treated mice. $\mathrm{CXB}$, celecoxib; TRAIL, tumor necrosis factor-related apoptosis-inducing ligand.

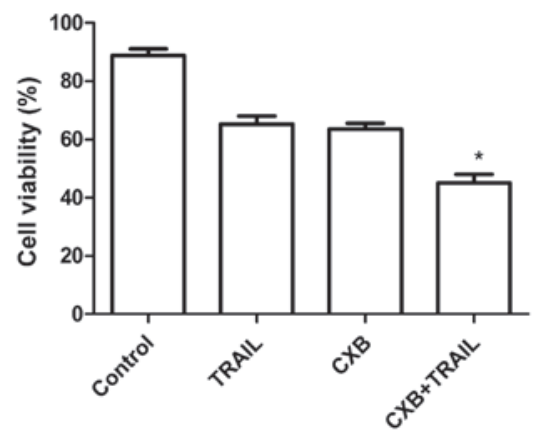

Figure 4. MTT proliferation assay of mouse splenocytes. Data indicate mean \pm standard deviation of three independent experiments. ${ }^{*} \mathrm{P}<0.05$ compared with untreated mice. CXB, celecoxib; TRAIL, tumor necrosis factor-related apoptosis-inducing ligand.

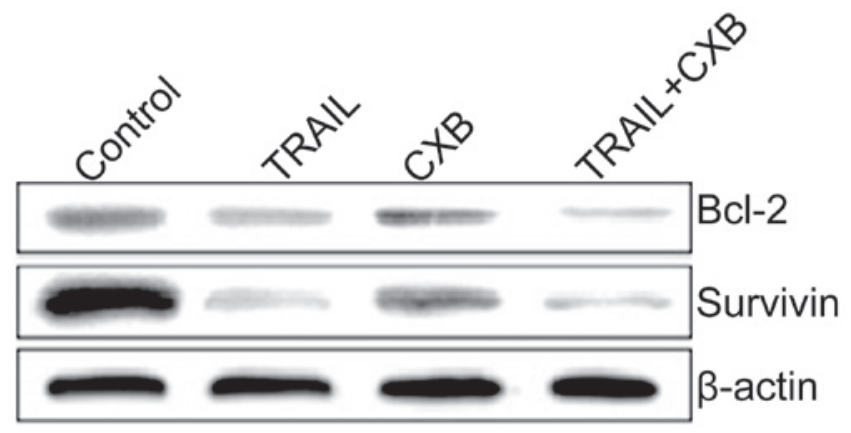

Figure 5. Protein expression profiles of MG-63 osteosarcoma cancer cells treated with TRAIL and/or CXB. The expression levels of the apoptotic proteins Blc-2 and survivin were determined by western blot analysis using the respective specific antibodies. $\beta$-actin served as an invariant control for equal loading. CXB, celecoxib; TRAIL, tumor necrosis factor-related apoptosis-inducing ligand; Bcl-2, B-cell lymphoma 2.

first to reveal that combining clinical doses of TRAIL with CXB reduces OS tumor cell apoptosis and inhibits OS tumor growth in vivo.

Amongst the numerous methods to stimulate tumor cell apoptosis, induction of the death receptor ligand TRAIL shows particular promise. Previous preclinical studies have observed that TRAIL induces tumor cell apoptosis in vivo without causing lethal toxicity $(23,24)$. One main issue in the clinical use of TRAIL is its limited efficacy in monotherapeutic approaches in different tumor entities. Thus, investigation into methods that enhance the capacity of TRAIL to induce apoptosis is required.

Recent preclinical studies have observed that TRAIL efficacy is increased through the use of combined chemotherapy $(25,26)$. Furthermore, a phase Ib trial was conducted with rhTRAIL administered in combination with rituximab in patients with low-grade non-Hodgkin lymphoma. Gaiser et al (27) demonstrated that CXB suppressed survivin levels by proteasomal degradation and thereby induced apoptosis and enhanced TRAIL-mediated cytotoxicity in U87, U251 and A172 glioma cells. In addition, Lu et al (28) observed that $\mathrm{CXB}$ and camptothecin treatment sensitized TRAIL-resistant HepG2 and Hep3B hepatocellular carcinoma (HCC) cell lines to TRAIL-induced apoptosis through downregulation of cellular Fas-associated death domain-like interleukin- $1 \beta$-converting enzyme-inhibitory protein and cleavage of caspase- 8 and caspase- 3 . These studies demonstrated that combination therapy with TRAIL and other anticancer drugs significantly inhibited cell viability compared with single drug treatments. The present study revealed that combining TRAIL with CXB significantly induced cell apoptosis and inhibited OS tumor growth, whereas single drug treatment did not exert statistically significant effects. These results further demonstrated that combination treatments in cancer therapy may be more effective than single drug treatments.

In combined chemotherapy, drugs with different mechanisms of action are employed simultaneously, reducing the possibility that resistant cancer cells survive and proliferate. When drugs exerting different effects are combined, each drug may be used at the optimal dose, without intolerable side effects (29). Numerous studies have demonstrated that the anticancer activity of standard chemotherapeutic agents is enhanced by the addition of CXB (30). The data from the present study revealed that CXB sensitized TRAIL-resistant MG-63 OS cell lines to TRAIL-induced apoptosis through downregulation of cellular Bcl-2 and survivin expression. These results further confirmed that the administration of $\mathrm{CXB}$ in combination with other anticancer drugs improves the antitumor effect.

In conclusion, the present study demonstrated that combining TRAIL with CXB significantly induced cell apop- 
tosis and inhibited OS tumor growth in a nude rat model. This combination regimen requires further evaluation in clinical trials, following further preclinical studies.

\section{Acknowledgements}

This study was supported by the Science and Technology Research and Innovation Team funded by Jilin Province (grant no. JL2011088).

\section{References}

1. Caronia D, Patiño-Garcia A, Peréz-Martínez A, et al: Effect of $\mathrm{ABCB} 1$ and $\mathrm{ABCC} 3$ polymorphisms on osteosarcoma survival after chemotherapy: a pharmacogenetic study. PLoS One 6: e26091, 2011.

2. Bacci G, Balladelli A, Palmerini E, et al: Neoadjuvant chemotherapy for osteosarcoma of the extremities in preadolescent patients: the Rizzoli Institute experience. J Pediatr Hemato Oncol 30: 908-912, 2008.

3. Ta HT, Dass CR, Choong PF and Dunstan DE: Osteosarcoma treatment: state of the art. Cancer Metastasis Rev 28: 247-263, 2009.

4. Gorlick R, Anderson P, Andrulis I, et al: Biology of childhood osteogenic sarcoma and potential targets for therapeutic development: meeting summary. Clin Cancer Res 9: 5442-5453, 2003.

5. Mérino D, Lalaoui N, Morizot A, Solary E and Micheau O: TRAIL in cancer therapy: present and future challenges. Expert Opin Ther Targets 11: 1299-1314, 2007.

6. Johnstone RW, Frew AJ and Smyth MJ: The TRAIL apoptotic pathway in cancer onset, progression and therapy. Nat Rev Cancer 8: 782-798, 2008.

7. Almasan A and Ashkenazi A: Apo2L/TRAIL: apoptosis signaling, biology, and potential for cancer therapy. Cytokine Growth Factor Rev 14: 337-348, 2003.

8. Ashkenazi A, Holland P and Eckhardt SG: Ligand-based targeting of apoptosis in cancer: the potential of recombinant human apoptosis ligand 2/Tumor necrosis factor-related apoptosis-inducing ligand (rhApo2L/TRAIL). J Clin Oncol 26: 3621-3630, 2008.

9. Walczak H, Miller RE, Ariail K, et al: Tumoricidal activity of tumor necrosis factor-related apoptosis-inducing ligand in vivo. Nat Med 5: 157-163, 1999.

10. Ganten TM, Haas TL, Sykora J, et al: Enhanced caspase-8 recruitment to and activation at the DISC is critical for sensitisation of human hepatocellular carcinoma cells to TRAIL-induced apoptosis by chemotherapeutic drugs. Cell Death Differ 11 Suppl 1: S86-S96, 2004.

11. Gómez-Benito M, Martinez-Lorenzo MJ, Anel A, Marzo I and Naval J: Membrane expression of DR4, DR5 and caspase-8 levels, but not Mcl-1, determine sensitivity of human myeloma cells to Apo2L/TRAIL. Exp Cell Res 313: 2378-2388, 2007.

12. Schuchmann M, Schulze-Bergkamen H, Fleischer B, et al: Histone deacetylase inhibition by valproic acid down-regulates c-FLIP/CASH and sensitizes hepatoma cells towards CD95-and TRAIL receptor-mediated apoptosis and chemotherapy. Oncol Rep 15: 227-230, 2006.

13. He SQ, Rehman H, Gong MG, et al: Inhibiting survivin expression enhances TRAIL-induced tumoricidal activity in human hepatocellular carcinoma via cell cycle arrest. Cancer Biol Ther 6: $1247-1257,2007$
14. Han J, Goldstein LA, Gastman BR and Rabinowich H: Interrelated roles for Mcl-1 and BIM in regulation of TRAIL-mediated mitochondrial apoptosis. J Biol Chem 281: 10153-10163, 2006.

15. Bortul R, Tazzari PL, Cappellini A, et al: Constitutively active Akt1 protects HL60 leukemia cells from TRAIL-induced apoptosis through a mechanism involving NF-kappaB activation and cFLIP(L) up-regulation. Leukemia 17: 379-389, 2003.

16. Bosch FX, Ribes J and Borras J: Epidemiology of primary liver cancer. Semin Liver Dis 19: 271-285, 1999.

17. Winfield LL and Payton-Stewart F: Celecoxib and Bcl-2: emerging possibilities for anticancer drug design. Future Med Chem 4: 361-383, 2012.

18. Wei D, Wang L, He Y, et al: Celecoxib inhibits vascular endothelial growth factor expression in and reduces angiogenesis and metastasis of human pancreatic cancer via suppression of Sp1 transcription factor activity. Cancer Res 64: 2030-2038, 2004.

19. Jeon YW and Suh YJ: Synergistic apoptotic effect of celecoxib and luteolin on breast cancer cells. Oncol Rep 29: 819-825, 2013.

20. Mikuła-Pietrasik J, Kuczmarska A, Kucińska M, et al: Resveratrol and its synthetic derivatives exert opposite effects on mesothelial cell-dependent angiogenesis via modulating secretion of VEGF and IL-8/CXCL8. Angiogenesis 15: 361-376, 2012.

21. Yoshida S, Amano H, Hayashi I, et al: COX-2/VEGF-dependent facilitation of tumor-associated angiogenesis and tumor growth in vivo. Lab Invest 83: 1385-1394, 2003.

22. Zhang X, Zou Y, Mao Y, et al: Preclinical toxicity of DATR, a recombinant soluble human TRAIL mutant, in rats and cynomolgus monkeys. Regul Toxicol Pharmacol 61: 230-235, 2011.

23. Ashkenazi A: Targeting death and decoy receptors of the tumour-necrosis factor superfamily. Nat Rev Cancer 2: 420-430, 2002.

24. Koschny R, Walczak H and Ganten TM: The promise of TRAIL - potential and risks of a novel anticancer therapy. J Mol Med (Berl) 85: 923-935, 2007.

25. Cuello M, Ettenberg SA, Nau MM and Lipkowitz S: Synergistic induction of apoptosis by the combination of trail and chemotherapy in chemoresistant ovarian cancer cells. Gynecol Oncol 81: 380-390, 2001.

26. Nagane M, Pan G, Weddle JJ, et al: Increased death receptor 5 expression by chemotherapeutic agents in human gliomas causes synergistic cytotoxicity with tumor necrosis factor-related apoptosis-inducing ligand in vitro and in vivo. Cancer Res 60: 847-853, 2000.

27. Gaiser T, Becker MR, Habel A, et al: TRAIL-mediated apoptosis in malignant glioma cells is augmented by celecoxib through proteasomal degradation of survivin. Neurosci Lett 442: 109-113, 2008.

28. Lu G, Liu Y, Ji B, et al: Synergistic effect of celecoxib on TRAIL-induced apoptosis in hepatocellular carcinoma cells. Cancer Invest 28: 629-634, 2010.

29. Ullah MF: Cancer multidrug resistance (MDR): a major impediment to effective chemotherapy. Asian Pac J Cancer Prev 9: 1-6, 2008.

30. Irie T, Tsujii M, Tsuji S, et al: Synergistic antitumor effects of celecoxib with 5-fluorouracil depend on IFN-gamma. Int J Cancer 121: 878-883, 2007. 